\title{
Technical Analysis of FDM Color 3D Printing Nozzle
}

\author{
Jinxian $\mathrm{Qi}^{1,2, \mathrm{a}^{*}}$, Chong $\operatorname{Tan}^{1,2, \mathrm{~b}}$ and Cuiqiao $\mathrm{Li}^{1,2, \mathrm{c}}$
}

${ }^{1}$ Key Laboratory of Metallurgical Equipment and Control Technology, Ministry of Education,

Wuhan University of Science and Technology, Wuhan 430081, China.

${ }^{2}$ Hubei Key Laboratory of Mechanical Transmission and Manufacturing Engineering,

Wuhan University of Science and Technology, Wuhan 430081, China.

a1847820924@qq.com, ${ }^{b}$ tc18983100080@163.com, ${ }^{\circ} 1137410667 @ q q . c o m$

Keywords: FDM color 3D printing; Print nozzle structure analysis; No boosting and boosting three-way mixing nozzle

\begin{abstract}
In recent years, 3D printing technology has been used in all walks of life because of the rapid development of $3 \mathrm{D}$ printing technology. Due to the pursuit of personalization, color 3D printing technology has developed rapidly since its appearance. This paper first summarizes the research development and application status of FDM color 3D printing. Starting with the printing process and technical principle of FDM color 3D printing technology, the shortages and main contradictions of the existing color printing nozzles are summarized. The design principle, structure, merits and demerits of the multi-color printing nozzle are analyzed, and some improvements are proposed for the current nozzle. The goal is to take color 3D printing a step further.
\end{abstract}

\section{Introduction}

3D printing technology was proposed in the 90s of last century, has now become an emerging manufacturing technology and rapid development. 3D printing technology is also called Additive Manu-facturing (AM). 3D printing technology has rapidly penetrated into fields such as medicine, aerospace, automobile industry, culture and entertainment, food processing and model making[1][2][3]. Color printing than monochrome printing based on advantages, more able to adapt to diverse social needs. The combination of FDM and subtractive color mixing constitutes the principle of color $3 \mathrm{D}$ printing in which different colored materials are mixed to a specific mixed color in a certain ratio. The nozzle, as the main executive component of the color $3 \mathrm{D}$ printer function, is a highly integrated part that mainly finishes the work of melting the wire, mixing evenly and depositing prints.

\section{The Development and Current Situation of FDM 3D Printing}

James Corbett [4] made a more detailed study of color mixing nozzles. For the first time, he gave several nozzle mechanisms. Then, taking the static mixer of glue as a control, he compared several hybrid nozzles with different structures. But the internal structure of these nozzles are complex.

A foreign 3D printer company Builder released a new colomixing 3D printing machine. The printer is equipped with a hybrid print nozzle. This $\mathrm{G}$ code with color information is generated by a blending generator application that allows customers to choose the mix of colors for their own.

Forest 3D Printing Technology Company in Shenzhen of China, has released a Color Mixer 3D Printer MakerPi M2030X, which uses a three-primary color principle to achieve color printing through the blending of multicolor materials too. 


\section{FDM-Type Color Printing Principle and Process}

The biggest difference between color printing and monochrome printing based on FDM technology is the generation of nozzle structure and G-code, as shown in figure 1. Color printing nozzle to take

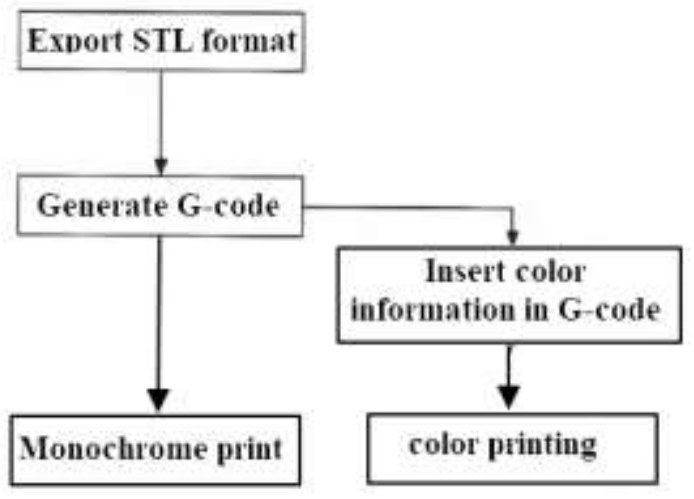

Fig.1 Monochrome printing and color printing process

dual color mixing nozzle, three color mixing nozzle or multi-color mixing nozzle. This kind of nozzle plays a role of mixing molten material. Because G-code of color printing contains the color information for each slice layer, there is a big difference in how G-code is generated.

Color printing color mixing principle. Color printing principle is the subtractive color mixing method [5], the subtractive color mixing method of the three primary colors Cyan, Magenta and Yellow, also known as CMY. The general color 3D printing using these three colors of the material, after melting in accordance with the different percentages can be mixed to get a specific color

Color printing process. Color printing process can be divided into three steps which are the print model data acquisition, model data processing and color printing.

Firstly, the print model data acquisition. There are usually two options to create a model, using 3D software such as Inventor, UG, or reverse scanning through 3D scanner to acquire 3D model. Secondly, model data processing [6] [7]. The model is converted to STL format. Then the model will be sliced. After that, the smoothing needs to be done to make each layer of the printed model to be over smoother. Some three-dimensional models require additional support structure when printing, so as to keep the model geometry during deposition printing Stability [8]. Than is the insertion of color information to get the $\mathrm{G}$ code with color information. Finally, color printing. The feeding system controls the feeding speed of different material wires, so as to achieve the purpose of printing a specific color. The material in the nozzle is melted at high temperature and mixed into the mixing chamber through the drainage chamber under the pressure of the rear. The materials of different colors are fully mixed and deposited and printed after being extruded.

\section{Print Nozzle Structure Analysis}

Based on the principle of multi-color mixing, the current FDM-type color printer extrusion mechanism is to take more into the single out of the way, by controlling the speed of different colors into the wire, to achieve different proportions of the mix, resulting in a rich color.

Shortcomings of current color 3D printing nozzle. Firstly no uniform temperature distribution inside the nozzle [9]. FDM 3D printing materials are thermoplastic polymer compounds, melting at high temperatures to viscous flow state. During the whole process of entering the material into the extrusion, it will pass through different areas of the nozzle. The temperature in these areas is not consistent. The change of the whole process temperature will cause the melt to expand or contract, which will affect the flow rate and pressure of melt eventually lead to a decrease in the quality of the printed product. 
Secondly extruded spinneret pressure is not stable. Because the control system accuracy is not enough and each color of the material into the speed changes directly affect the melting chamber, the drainage chamber and the mixing chamber pressure, thus affecting the extrusion speed of the material, will lead to the accumulation of wire material accumulation, the nozzle plug the phenomenon, the impact product printing accuracy.

Finally the conflict of the color accuracy and uniformity can't be solved [10]. The mixing chamber determines the color accuracy and uniformity. When the mixing chamber is reduced, different color materials in the mixing chamber of the micro-volume can't be uniformly mixed, but the accuracy of the color will increase. Conversely, the uniformity will drop, the color accuracy increases. the uniformity will drop, the color accuracy increases.

The theoretical basis of nozzle design. C. Ren [9] et al. analyzed the fluidity and thermal balance of the melt inside the nozzle. The flow field of the nozzles with different diameters will make the pressure difference of each flow channel. Based on the analysis of the flow paths of the circular cross section and the tapered circular pipe, the total pressure difference (refer with Eq.1)

$$
\Delta \mathrm{P}=\nabla P_{1}+\nabla P_{2}+\nabla P_{3}=\frac{K_{P} Q^{n}}{D_{1}^{3 n+1}}\left[L_{1}+\frac{g\left(k_{d}\right)}{3 n} L_{2}+K_{D}^{3 n+1} L_{3}\right]
$$

The formula provides a reliable theoretical basis for the internal flow channel design of the nozzle. The internal temperature of the nozzle has a great influence on the flow rate of the melt, the pressure change and the temperature of the melt, and the change of the temperature has a great influence on the various effects caused by the elasticity of the material inside the nozzle. According to the heat balance equation (refer with Eq.2)

$$
\left(Q_{M E}+Q_{H}\right) \cdot\left(Q_{M A}+Q_{C A}+Q_{R A D}+Q_{h}\right)=\frac{\partial}{\partial t}\left(m_{d} \cdot C_{p d} \cdot T_{d}\right)
$$

Under the stable working condition, the right part is zero, and the temperature rise of the molten body is only related to the pressure loss of the nozzle and the material of the molten body.

S.L. Han [11] used UG finite element module, a dual color mixing nozzle temperature field simulation analysis. L. Xiao [12] and others also conducted a thermodynamic analysis of the nozzle. The analysis is mainly aimed at the poor precision caused by the unreasonable thermal structure of the print nozzle and the corresponding improvement method is put forward. This resulted in a $12.5 \%$ reduction in the deformation of the nozzle, while the overall temperature distribution tended to be reasonable.

S.B. Wang [13] designed and simulated several dual color mixing nozzles and three color mixing nozzles, got a variety of color mixing nozzle in the normal circumstances when the flow of molten silk flow resistance, heating rod heating energy utilization and the warm-up time of color mixing nozzle heated from room temperature to the working state.

Improve the structure of the nozzle. Sprinklers are the core components of the extrusion mechanism, which plays an important role. The structure of the nozzle has a crucial impact on print quality.[13]

No boosting three-way mixing nozzle [5], as shown in Fig.2. 


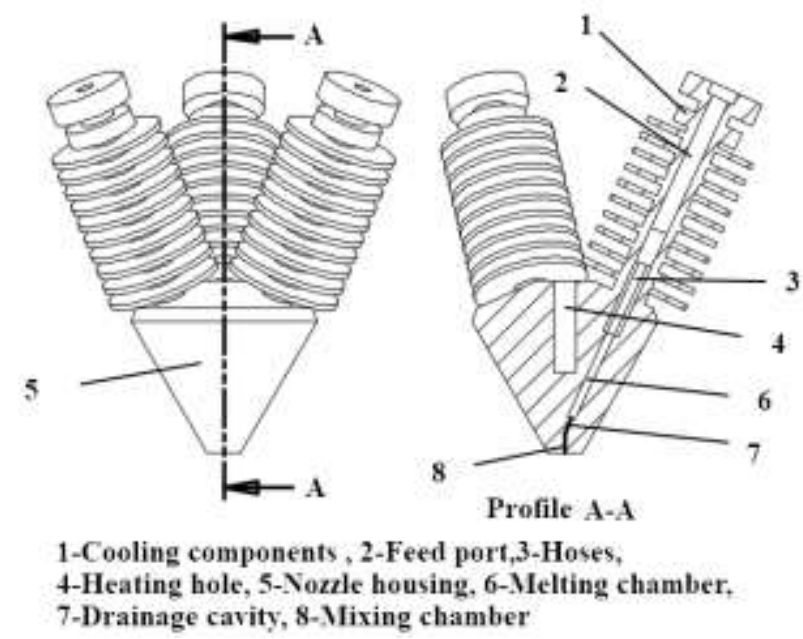

Fig. 2 No boosting three-way mixing nozzle

The main components of the color 3D printer nozzle are feed port, cooling components, nozzle mouth, heating hole. The nozzle mouth has a melting chamber, a drainage chamber and a mixing chamber. This nozzle drainage chamber, the volume of the mixing chamber are relatively small, can effectively solve the problem of uneven color. In addition, with a simple structure, the response time is a bit short, can print a distinctive color of the parts. Due to the bottleneck between the mixing chamber and the drainage chamber, the flow of molten material creates a pressure differential and temperature changes that affect its print quality.

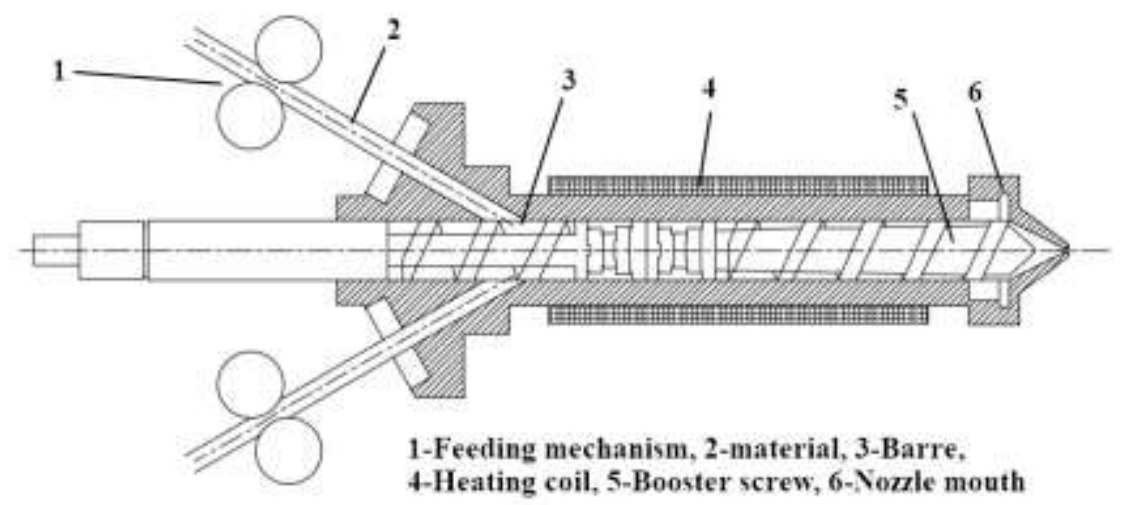

Fig.3 Boosting three-way mixing nozzle

Boosting three-way mixing nozzle [15], as shown in Fig.3, adopted a screw boost method. The main structure of a nozzle mouth, barrel, heating coil, extrusion screw, feeding agencies The advantage of this structure is that the heating coil is completely wrapped on the outside of the spray head so that the whole spray head can be uniformly heated. The temperature of the melt chamber and the mixing chamber of the spray head can be kept consistent. Booster screw with a stable pressure and flow rate role. The biggest drawback is that the melting chamber and the mixing chamber merge together so that the entire interior space becomes larger. The response time that resulted in a change in the print color was increased.

\section{Summary}

This paper mainly introduces the development history of FDM type color 3D printer, the technical theory of color printing and the color printing nozzle, and summarizes and discusses the design principle of the current color 3D print head and draw the main problems of the current print head. Based on the above problems and contradictions, this paper summarizes some theories and solutions, and analyzes two typical three-way hybrid printing nozzle structure. It provides a good basis for the 
design and use of nozzle device. Color 3D printing has entered a phase of rapid growth, but how to use FDM-based printers to print more efficiently and more accurately color 3D products still need to be further explored.

\section{References}

[1] L.X. Zhou, X.K. Chen and X. Wu: Technology Innovation and Application, Vol. 28 (2017) No.28, P.28

[2] X.L. Li, J.X. Ma, P. Li, Q. Chen and W.M. Zhou: Process Automation Instrumentation, Vol. 35 (2014) No.1, p.1

[3] Y.S. Shi, L.C. Zhang, Y. Bai and Z.Y. Zhao: Scientia Sinica (Informationis), Vol. 45 (2015) No.2, p.197

[4] James Corbett: Reprap colour mixing project (MS., University of Bath, United Kingdom 2012), p. 1

[5] Z.F. Huang, Y.L. Ma, J.M. Li, O.Y. Li and J. Liu: Machine Tool \& Hydraulics, Vol. 45 (2017) No.4, p. 21

[6] B.X. Shen and Y.P. Guan: Journal of Beijing Information Science \& Technology University, Vol. 31 (2016) No.5, p.60

[7] C Andrej, P Vojko and S Zoran: International Journal of Information and Computer Science, (2015) No.4, p.1

[8] H Rezayat, W Zhao and A Siriruk: material science and technology,Vol.31 (2015) No.8, p.894

[9] C. Ren, J. Huang and C.L. Guan: Mechanical Research \& Application, Vol. 27 (2014) No.1, p.62

[10]Z.H. Cai and G.H. Zhu: Heilongjiang Science, Vol. 7 (2016) No.22, p.16

[11] S.L. Han, Z.Y. Li, Y. Xiao and X. Xu: Machinery Design \& Manufacture, (2015) No.11, p.116

[12] L. Xiao, X.M. Ma, Y.Y. Yao and Z.Y. Xie: Machinery, Vol.52 (2015) No.599, p.15

[13] S.B. Wang: CFD Analysis of Fused Deposition Mopdelling Nozzles and Optimal Design of mixing Nozzles (Ms., Harbin Institute of Technology, China 2016), p.1

[14] Adam Kłodowski, H Eskelinen and AS Semken: Robotica, Vol.33 (2014) No.4, p.721

[15] Q.Y. Sun: China Patent 204585854U. (2015) 